\title{
DOI:https://doi.org/10.24297/jam.v19i.8896
}

\section{Partition Theoretic Interpretation of Two Identities of Euler}

Ananta Kr. Bora.

Concept Jr. College, Assam (India)

Email:anantakrbora.bora610@gmail.com

\section{Abstract:}

In this paper we have derived generating function for a restricted partition function. This is in conjunction two identities of Euler provides new partition theoretic interpretation of two identities of Euler.

\section{Introduction , Definition and the Main Results}

The following two " Sum -Product" Identities are known as Rogers - Ramanujan identities :

$$
\begin{aligned}
& \sum_{n=0}^{\infty} \frac{q^{n^{2}}}{(q ; q)_{n}}=\prod_{n=1}^{\infty} \frac{1}{\left(1-q^{5 n-1}\right)\left(1-q^{5 n-4}\right)} \\
& \sum_{n=0}^{\infty} \frac{q^{n^{2}+n}}{(q ; q)_{n}}=\prod_{n=1}^{\infty} \frac{1}{\left(1-q^{5 n-2}\right)\left(1-q^{5 n-3}\right)}
\end{aligned}
$$

Where $|q|<1$ and $(q ; q)_{n}$ is a rising factorial defined by

$$
(a ; q)_{n}=\prod_{n=0}^{\infty} \frac{1-a q^{i}}{1-a q^{n+i}}
$$

If $\mathrm{n}$ is a positive integer, then obviously

$$
\begin{gathered}
(a ; q)_{n}=(1-a)(1-a q)\left(1-a q^{2}\right) \ldots \ldots\left(1-a q^{n-1}\right) \\
(a ; q)_{\infty}=(1-a)(1-a q)\left(1-a q^{2}\right) \ldots \quad \ldots \quad \ldots
\end{gathered}
$$

In this paper we give the partition theoretic interpretation of the following two identities of Euler:

$$
\begin{aligned}
& \sum_{n=0}^{\infty} \frac{q^{n^{2}}}{\left(q^{2} ; q^{2}\right)_{n}}=\left(-q ; q^{2}\right)_{\infty} \\
& \sum_{n=0}^{\infty} \frac{q^{n(n+1)}}{\left(q^{2} ; q^{2}\right)_{n}}=\left(-q^{2} ; q^{2}\right)_{\infty}
\end{aligned}
$$

Theorem1 : For a positive integer $\mathrm{k}$, let $A_{k}(n)$ denote the number of partition of $\mathrm{n}$ such that the smallest part (or the only part $)$ is $\equiv k(\bmod 2)$ and the difference between any two parts is

$\equiv 0(\bmod 2)$ then

$$
\sum_{n=0}^{\infty} A_{k}(n)=\sum_{n=0}^{\infty} \frac{q^{n(n+k-1)}}{\left(q^{2} ; q^{2}\right)_{n}}
$$


Proof : Let $A_{k}(m, n)$ denote the number of partitions of $n$ enumerated by $A_{k}(n)$ into $m$ parts. We shall first show that

$$
A_{k}(m, n)=A_{k}(m-1, n-k-2(m-1))+A_{k}(m, n-2 m)
$$

To prove the identity (4) we split the partitions enumerated by $A_{k}(m, n)$ into two classes :

(i) those who have least part $k$

(ii) those who have least part greater than $k$

For those whose smallest part is equal to $k$, we delete $k$ and then subtract 2 from all the remaining parts. This produces a partition of $n-k-2(m-1)$ into exactly $m-1$ parts.

Those who have smallest part greater than $k$, we subtract 2 from each part that produced a partition of $n-2 m$ into $m$ parts. The transformations are invertible and thus we have

$A_{k}(m, n)=A_{k}(m-1, n-k-2(m-1))+A_{k}(m, n-2 m)$

For $|q|<1$ and $|z q|<1$, let

$$
f_{k}(z, q)=\sum_{n=0}^{\infty} \sum_{m=0}^{\infty} A_{k}(m, n) z^{m} q^{n}
$$

Substituting $A_{k}(m, n)$ from (4)in (3)and then simplifying, we get

$$
f_{k}(z, q)=z q^{k} f_{k}\left(z q^{2}, q\right)+f_{k}\left(z q^{2}, q\right) \quad \ldots \ldots
$$

Setting

$$
f_{k}(z, q)=\sum_{n=0}^{\infty} \alpha(n, k ; q) z^{n} \quad \text { and then comparing the coefficients of } z^{n} \text { on both sides of (6) we }
$$

see that

$$
\alpha(n, k ; q)=\frac{q^{2 n-1+k}}{1-q^{2 n}} \alpha(n-1, k ; q) \quad \ldots \quad \ldots
$$

Iterating (6), $\mathrm{n}$ times and observing that $\alpha(0, k ; q)=1$, we see that

$$
\begin{gathered}
\alpha(n, k ; q)=\frac{q^{n(n+k-1)}}{\left(q^{2} ; q^{2}\right)_{n}} \\
\therefore \quad f_{k}(z, q)=\sum_{n=0}^{\infty} \alpha(n, k ; q) z^{n} \\
=\sum_{n=0}^{\infty} \frac{q^{n(n+k-1)}}{\left(q^{2} ; q^{2}\right)_{n}} z^{n} \\
=f_{k}(1, q) \\
=\sum_{n=0}^{\infty} \frac{q^{n(n+k-1)}}{\left(q^{2} ; q^{2}\right)_{n}}
\end{gathered}
$$


This completes the proof of Theorem1.

Particular Cases :

For $k=1$, theorem 1 reduces to the identity (1)

For $\mathrm{k}=2$, theorem reduces to the identity (2)

\section{References :}

1. L.J. Rogers," Second memoir on the expansion of certain infinite products", Proc. Of London Math.Soc.vol 25 .pp $318-343,1894$

2. P.A.MacMohan, Combinatory Analysis, Vol2 . Cambridge University Press, New York ,USA, 1916.

3. B. Gordon, "A combinatorial generalization of the Rogers - Ramanujan identities," Amer.Jour. of Math. Vol . 83 .pp 393-399, 1961.

4. A.K.Agarwal and M.Goyal , "New Partition Theoritic Interpretations of Rogers - Ramanujan Identities," International Journal of combinatorics.Vol.2012,Article ID 409505, Hindawi Publishing Corporation.

5. B.Gordon ,"Some continued fractions of the Rogers - Ramanujan type,"Duke Mathematical Journal ,Vol32,pp.741-748,1965.

6. W.G.Connor, "Partition theorems related to some identities of Rogers and Watson," Transactions of the American Mathematical Society,Vol.214,pp 95-111,1975

7. M.D.Hirschhorn, "Some partition theorems of the Rogers-Ramanujan type,"Journal of Combinatorial theory,vol.27,no.1,pp.33-37,1979.

8. A.K.Agarwal and G.E.Andrews, "Hook differences and lattice paths'" Journal of Statistical Planning and Inference,vol.14,no.1,pp.5-14,1986. [9] m.V.Subbarao and A.K. agarwal, "Further theorems of the Rogers - Ramanujan type theorems,"

9. Canadian Mathematical Bulletin,vol.31,no.2,pp210-214, 1988. [10]D.M. Bressoud, "A new family of partition identities," Pacific Journal of Mathematics, vol.77,pp.71-74,1978. 